\title{
The Effect of Turbulence on Mixing in Prototype Reaction-Diffusion Systems
}

\author{
ANDREW MAJDA \\ Courant Institute \\ PANAGIOTIS SOUGANIDIS \\ University of Wisconsin-Madison
}

\begin{abstract}
The effect of turbulence on mixing in prototype reaction-diffusion systems is analyzed here in the special situation where the turbulence is modeled ideally with two separated scales consisting of a large-scale mean flow plus a small-scale spatiotemporal periodic flow. In the limit of fast reaction and slow diffusion, it is rigorously proved that the turbulence does not contribute to the location of the mixing zone in the limit and that this mixing zone location is determined solely by advection of the large-scale velocity field. This surprising result contrasts strongly with earlier work of the authors that always yields a large-scale propagation speed enhanced by small-scale turbulence for propagating fronts. The mathematical reasons for these differences are pointed out. This main theorem rigorously justifies the limit equilibrium approximations utilized in nonpremixed turbulent diffusion flames and condensation-evaporation modeling in cloud physics in the fast reaction limit. The subtle nature of this result is emphasized by explicit examples presented in the fast reaction and zero-diffusion limit with a nontrivial effect of turbulence on mixing in the limit. The situation with slow reaction and slow diffusion is also studied in the present work. Here the strong stirring by turbulence before significant reaction occurs necessarily leads to a homogenized limit with the strong mixing effects of turbulence expressed by a rigorous turbulent diffusivity modifying the reaction-diffusion equations. Physical examples from non-premixed turbulent combustion and cloud microphysics modeling are utilized throughout the paper to motivate and interpret the mathematical results. (c) 2000 John Wiley \& Sons, Inc.
\end{abstract}

\section{Introduction}

Reaction-diffusion-convection equations provide an important scientific description for diverse phenomena such as combustion [14, 24], transport in ecology [13], phase changes in atmospheric cloud dynamics [11, 12], and biologicalchemical transport in the ocean [2]. In all of the applications listed above, the convective transport typically has large-scale spatial components that are well-resolved and can be measured, as well as a significant velocity field contribution varying on 
smaller spatial scales, usually due to turbulence, which cannot be resolved in detail. The effect of such unresolved scales on the large-scale dynamics and mixing is an important practical issue.

Here the authors present a mathematically rigorous study of the effect of smallscale turbulence on mixing and large-scale dynamics for prototype reaction-diffusion systems. The techniques utilized are similar to those in earlier mathematically rigorous work of the authors assessing the effects of small-scale turbulence on front propagation for reaction-diffusion equations [4, 5, 6, 17, 18, 19]. However, the physical phenomena and mathematical results developed are completely different. In the context of combustion theory, the model problems studied here involve non-premixed turbulent diffusion flames, while the earlier studies by the authors involve the effects of turbulence on premixed flame propagation. To motivate the main results in this paper and to illustrate the important physical differences mentioned in the previous discussion, we next describe the simplest model treated here, namely, isothermal non-premixed turbulent diffusion flames.

Isothermal non-premixed turbulent combustion is characterized by two variables $Y_{F}$ and $Y_{\theta}$ representing the mass fraction of fuel and oxidizer, respectively. In non-premixed combustion, the fuel and oxidizer occupy different regions of space initially and are mixed to react together through advection by the fluid velocity field, which has both large-scale and turbulent components. With the assumptions that the fuel and oxidizer react through a simple single-step kinetics (see $[14,24]$ ), the mass fractions satisfy the coupled reaction-diffusion equations

$$
\left\{\begin{array}{l}
D_{t} Y_{F}=\mathcal{K} \Delta Y_{F}-K Y_{F} Y_{\theta} \\
D_{t} Y_{\theta}=\mathcal{K} \Delta Y_{\theta}-K Y_{F} Y_{\theta}
\end{array} \quad \text { in } \mathbb{R}^{N} \times(0, \infty),\right.
$$

where $\mathcal{K} \geq 0$ is the diffusion coefficient and $K>0$ is the reaction rate. The symbol $D_{t}$ denotes the convective derivative

$$
D_{t}=\partial_{t}+\vec{V} \cdot D
$$

with imposed velocity $\vec{V}$.

In non-premixed combustion, the fuel and oxidizer initially occupy disjoint regions of space; i.e., there are two disjoint sets $\Omega_{+}$and $\Omega_{-}$with $\Omega_{+} \cup \Omega_{-}=\mathbb{R}^{N}$ such that

$$
Y_{F}=\left\{\begin{array}{ll}
Y_{F}^{0}>0 & \text { on } \Omega_{+} \times\{0\} \\
Y_{F}^{0}=0 & \text { on } \Omega_{-} \times\{0\}
\end{array} \quad \text { and } \quad Y_{\theta}= \begin{cases}Y_{\theta}^{0}=0 & \text { on } \Omega_{+} \times\{0\} \\
Y_{\theta}^{0}>0 & \text { on } \Omega_{-} \times\{0\}\end{cases}\right.
$$

By introducing the variables

$$
Y=Y_{F}-Y_{\theta} \quad \text { and } \quad Z=Y_{F},
$$

the equations in (1.1) can be rewritten as

$$
\left\{\begin{array}{l}
D_{t} Z=\mathcal{K} \Delta Z+K Z(Y-Z) \\
D_{t} Y=\mathcal{K} \Delta Y
\end{array} \quad \text { in } \mathbb{R}^{N} \times(0, \infty),\right.
$$


with the initial data such that

$$
Z_{0}=\left\{\begin{array}{ll}
Y_{F}^{0} & \text { in } \Omega_{+} \times\{0\} \\
0 & \text { in } \Omega_{-} \times\{0\}
\end{array} \text { and } Y_{0}= \begin{cases}Y_{F}^{0} & \text { in } \Omega_{+} \times\{0\} \\
-Y_{\theta}^{0} & \text { in } \Omega_{-} \times\{0\}\end{cases}\right.
$$

The crucial feature for the initial data (1.6) in non-premixed turbulent combustion is the assumption that

the initial data describe different stable equilibria for the reaction in the regions $\Omega_{+}$and $\Omega_{-}$,

since we have

$$
\left.\frac{d}{d Z}\right|_{\left(Y_{0}^{ \pm}, Z_{0}^{ \pm}\right)}(Z(Y-Z))<0 .
$$

In contrast, for premixed turbulent combustion for the equations in (1.4), we have the "stoichiometric" balance condition $Y \equiv Y_{0}>0$ with $Y_{0}$ constant and the K-P-P equation for $Z$

$$
D_{t} Z=\mathcal{K} \Delta Z+K Z\left(Y_{0}-Z\right) \text { in } \mathbb{R}^{N} \times(0, \infty),
$$

with initial data

$$
Z= \begin{cases}Y_{0} & \text { in } \Omega_{+} \times\{0\} \\ 0 & \text { in } \Omega_{-} \times\{0\}\end{cases}
$$

In this situation the equilibrium in $\Omega_{+}$is stable, while the one in $\Omega_{-}$is unstable, leading to turbulence-enhanced front propagation in the fast reaction/slow diffusion limit $[4,5,6,8,9,10,17,18,19]$. We will see below that the phenomena for $(1.4)$ and (1.5) are very different.

To study the effects of turbulence on mixing for the problem in (1.4) and (1.5), we assume throughout this paper that the velocity field has the form

$$
\vec{V}(x, t)=\bar{V}(x, t)+v\left(\varepsilon^{-\alpha} x, \varepsilon^{-\alpha} t\right)
$$

where $\bar{V}$ is the large-scale flow and is a zero-mean space-time periodic velocity representing the effects of small-scale turbulence with $\varepsilon \ll 1$ a small parameter and the parameter $\alpha$ fixed with $0<\alpha \leq 1$. Thus, the velocity field satisfies the same hypotheses as in [17].

Next we describe the main results of this paper, specialized to the system in (1.4) and (1.5). Similar results remain valid for prototype turbulent reactiondiffusion equations of the form

$$
\left\{\begin{array}{l}
D_{t} \vec{Y}=\mathcal{K} \Delta \vec{Y} \\
D_{t} Z=\mathcal{K} \Delta Z+K Z(a(\vec{Y}, Z)-Z)
\end{array} \quad \text { in } \mathbb{R}^{N} \times(0, \infty)\right.
$$

with the reaction rate $K(\vec{Y}, Z)$ a suitable nonlinear function of $\vec{Y}$ and $Z$, where $\vec{Y}=$ $\left(\vec{Y}_{1}, \ldots, \vec{Y}_{m}\right)$ is a vector-valued function, and the initial data satisfying a natural generalization of (1.5) and (1.6). Physical examples, where $\vec{Y}$ is vector-valued and the nonlinear functions $K(\vec{Y}, Z)$ and $a(\vec{Y}, Z)$ actually occur, are presented in 
Section 2 and involve including the effects of temperature dependence from the reaction rate in $(1.1)$ to obtain $K(\vec{Y}, Z)$, while $a(\vec{Y}, Z)$ is typically nonlinear for the equations for bulk microphysics in cloud modeling [11, 12]. The statements for the theorems describing the behavior of the more general prototype systems in (1.10) can be found at the beginning of Sections 3, 4, and 5.

\subsection{The Effect of Turbulence on Mixing with Fast Reaction and Slow Diffusion}

In many applications of turbulent reaction-diffusion equations, it is natural to assume that the chemical reaction time is much shorter than the large-scale eddy turnover time, while the large-scale eddy turnover time is much shorter than the large-scale diffusion time. If the units of space and time are determined by the large-scale velocity field, the above requirements are satisfied by setting $K=$ $\varepsilon^{-1} K^{\prime}$ and $\mathcal{K}=\varepsilon \mathcal{K}^{\prime}$ in (1.4). Thus in this fast reaction/slow diffusion regime, the turbulent reaction-diffusion equations in (1.4) assume the form

$$
\left\{\begin{array}{l}
D_{t}^{\varepsilon} Y^{\varepsilon}=\varepsilon \mathcal{K} \Delta Y^{\varepsilon} \\
D_{t}^{\varepsilon} Z^{\varepsilon}=\varepsilon \mathcal{K} \Delta Z^{\varepsilon}+\varepsilon^{-1} K Z^{\varepsilon}\left(Y^{\varepsilon}-Z^{\varepsilon}\right)
\end{array} \quad \text { in } \mathbb{R}^{N} \times(0, \infty)\right.
$$

where the primes have been dropped in (1.11) for notational simplicity and we write $D_{t}^{\varepsilon}$ instead of $D_{t}$ to signify the dependence on $\varepsilon$ of the vector field. The structure for the small-scale velocity field in (1.9) represented by $v\left(\varepsilon^{-\alpha} x, \varepsilon^{-\alpha} t\right)$ has the following interpretation: As in actual turbulent flow fields, these velocities vary on smaller-length scales and evolve on faster time scales than the mean flow for any fixed $\alpha$ with $0<\alpha \leq 1$, and yet vary on at least as large a spatial scale as $0(\varepsilon)$, which can be regarded as the natural turbulent dissipation length scale in the model. These are the same scalings utilized in [17] to study turbulence-enhanced front propagation.

In contrast with [17], in this paper we prove the following:

THEOREM 1.1 Consider the solution $\left(Y^{\varepsilon}, Z^{\varepsilon}\right)$ of the reaction-diffusion system (1.11) with smooth initial data converging to the initial data $\left(Y_{0}, Z_{0}\right)$ defined in (1.5) and associated with two distinct stable equilibria, and assume that the velocity field $v$ is incompressible. Then, as $\varepsilon \rightarrow 0, Y^{\varepsilon} \rightarrow Y^{0}$, locally uniformly in $\mathbb{R}^{N} \times[0, \infty)$, and $Z^{\varepsilon} \rightarrow \max \left(Y^{0}, 0\right)$, locally uniformly in $\mathbb{R}^{N} \times(0, \infty)$, where $Y^{0}$ satisfies the large-scale advection equation

$$
Y_{t}^{0}+\bar{V} \cdot D Y^{0}=0 \quad \text { in } \mathbb{R}^{N} \times(0, \infty),
$$

with the initial data $\left.Y^{0}\right|_{t=0}=Y_{0}$. In particular, the small-scale velocity field does not affect the mixing zone in the limit and the mixing interface evolves with the large-scale flow.

As mentioned earlier, this result contrasts strongly with the results of $[4,5,6$, $17,18,19]$ for the situation in (1.7) where one region has an unstable equilibrium, 
where it is established that small-scale turbulence leads to enhanced effective largescale front propagation. The proof of Theorem 1.1 together with its more general version are presented in Section 3. Theorem 1.1 provides a rigorous justification for numerical algorithms that are based on the fast reaction limit and are utilized in numerical modeling of condensation-evaporation phenomena in cloud physics [12]. The theorem also provides a rigorous justification of the equilibrium chemistry approximation in the fast reaction limit for turbulent diffusion flames [14, 24] with the surprising additional fact that only the mean flow contributes to the location of the mixing zone in this limit.

\subsection{The Effect of Turbulence on Mixing with Fast Reaction and Zero Diffusion}

The result in Theorem 1.1 is subtle. In Section 4 we consider the limiting behavior as $\varepsilon \rightarrow 0$ of (1.11) with the initial data in (1.5) but with zero diffusion so that $\mathcal{K}=0$ for all $\varepsilon$. This is the fast reaction/zero diffusion limit. We obtain the following:

THEOREM 1.2 There are explicit examples of small-scale incompressible velocity fields $v$ such that the fast reaction/zero diffusion limit necessarily has nontrivial mixing by the turbulence and the mixing zone in the limit is not determined by the large-scale flow.

Thus Theorems 1.1 and 1.2 establish that turbulent mixing of a reactive scalar is very different in the fast reaction/slow diffusion and the fast reaction/zero diffusion limits. The explicit examples in Section 4 leading to Theorem 1.2 are based on earlier examples of Tartar [23] and E [3] for the limit of transport with oscillatory advection fields.

\subsection{Turbulent Mixing with Slow Reaction and Slow Diffusion}

In Section 5 we consider the situation with slow reaction and slow diffusion so that the reaction and diffusion terms in (1.4) both satisfy $\mathcal{K}=\varepsilon \mathcal{K}^{\prime}, K=\varepsilon K^{\prime}$. We also require that $\alpha=1$ for the turbulent velocity field in (1.9). For technical reasons, we also require that the mean flow $\bar{V}$ be relatively weak; i.e., it has the form

$$
\bar{V}=\varepsilon \bar{V}^{\prime}(x, \varepsilon t)
$$

Clearly very little chemical reaction occurs in this slow reaction/slow diffusion limit for order $O(1)$ times. With these assumptions, it is natural to look for mixing in the reaction-diffusion equations in (1.4) over the longer time scale $t^{\prime}=\varepsilon t$, where the reaction rates are not negligible. By introducing the primed variables in (1.4) and then dropping (for notational simplicity) the prime notation in the result, we 
obtain the following reaction-diffusion system:

$$
\begin{aligned}
& \left\{\begin{array}{l}
Y_{t}^{\varepsilon}+\left(\bar{V}(x, t)+\varepsilon^{-1} v\left(\varepsilon^{-1} x, \varepsilon^{-2} t\right)\right) \cdot D Y^{\varepsilon}=\mathcal{K} \Delta Y^{\varepsilon} \\
Z_{t}^{\varepsilon}+\left(\bar{V}(x, t)+\varepsilon^{-1} v\left(\varepsilon^{-1} x, \varepsilon^{-2} t\right) \cdot D Z^{\varepsilon}\right)=\mathcal{K} \Delta Z^{\varepsilon}+K\left(Z^{\varepsilon}\left(Y^{\varepsilon}-Z^{\varepsilon}\right)\right)
\end{array}\right. \\
& \text { in } \mathbb{R}^{N} \times(0, \infty) .
\end{aligned}
$$

Notice from (1.14) that the small-scale turbulence strongly stirs the fluid before significant chemical reaction occurs, so we can expect strong mixing effects of turbulence in the limit. In Section 5 we sketch the formal proof of the following result confirming this intuition:

THEOREM 1.3 Assume that the velocity field $v$ is incompressible. Then for any bounded initial data $\left(Y_{0}, Z_{0}\right)$ and any $T>0$, the solution $\left(Y^{\varepsilon}, Z^{\varepsilon}\right)$ of (1.14) converges locally uniformly in $\mathbb{R}^{N} \times(0, T)$, as $\varepsilon \rightarrow 0$, to the homogenized limit $(Y, Z)$ that satisfies the equations

$$
\left\{\begin{array}{l}
Y_{t}+\bar{V}(x, t) \cdot D Y=\mathcal{K} \Delta Y+D \cdot\left(\mathcal{K}^{*} D Y\right), \\
Z_{t}+\bar{V}(x, t) \cdot D Z=\mathcal{K} \Delta Z+D \cdot\left(\mathcal{K}^{*} D Z\right)+K Z(Y-Z)
\end{array} \quad \text { in } \mathbb{R}^{N} \times(0, \infty)\right.
$$

with the same initial data $\left(Y_{0}, Z_{0}\right)$.

The enhanced diffusivity tensor $\mathcal{K}^{*}$ is computed through the standard cell problem for homogenization of turbulent diffusion $[1,15,16,20]$. Finally, we note that this theorem applies for any initial data.

\section{Examples of Prototype Reaction Diffusion Equations in Non-Premixed Combustion and Cloud Physics}

We begin with the simplest model for non-premixed turbulent combustion that includes thermal effects, i.e., the release of heat through chemical reaction of the fuel and oxidizer and the nonlinear dependence of the reaction rate $K=K(T)$ in (1.1) on temperature. With these assumptions and the additional condition that the Lewis number is 1 , we obtain (see, for example, $[14,24]$ ) the following system of reaction-diffusion equations generalizing those in (1.1):

$$
\left\{\begin{array}{l}
D_{t} Y_{F}=\mathcal{K} \Delta Y_{F}-K(T) Y_{F} Y_{\theta} \\
D_{t} Y_{\theta}=\mathcal{K} \Delta Y_{\theta}-K(T) Y_{F} Y_{\theta} \quad \text { in } \mathbb{R}^{N} \times(0, \infty), \\
D_{t} T=\mathcal{K} \Delta T+q_{0} K(T) Y_{F} Y_{\theta}
\end{array}\right.
$$

where the constant $q_{0}>0$ measures the heat release due to chemical reaction. In order to ignore the feedback onto the actual fluid velocities, this heat release $q_{0}$ should be small.

In standard fashion, we introduce, in addition to the variables $Y=Y_{F}-Y_{\theta}$ and $Z=Y_{F}$ from (1.3), the enthalpy

$$
H=T+q_{0} Z,
$$


so that the system in (2.1) has the equivalent form

$$
\left\{\begin{array}{l}
D_{t} H=\mathcal{K} \Delta H \\
D_{t} Y=\mathcal{K} \Delta Y \\
D_{t} Z=\mathcal{K} \Delta Z+K\left(H-q_{0} Z\right) Z(Y-Z)
\end{array} \quad \text { in } \mathbb{R}^{N} \times(0, \infty) .\right.
$$

For non-premixed combustion, we have initial data for $Y, Z$ in equilibrium as in (1.5) and additional initial datum for $H$ denoted by $H_{0}^{ \pm}$such that the equilibria for the chemical reaction terms in $\Omega_{ \pm}$are both stable, i.e.,

$$
\left.\frac{d}{d Z}\right|_{\left(Y_{0}^{ \pm}, H_{0}^{ \pm}, Z_{0}^{ \pm}\right)} K\left(H-q_{0} Z\right) Z(Y-Z)<0 .
$$

The reader can easily check that the initial data in (1.5) for $\left(Y_{0}, Z_{0}\right)$ automatically satisfies the stability criterion in (2.3) for any nonlinear function $K(T)$. With the variables $\vec{Y}=(Y, H)$ and $Z$, we obtain a prototype reaction-diffusion system with the structure in (1.10), where the reaction term $K(\vec{Y}, Z)$ is nonlinear, but $a(\vec{Y}, Z)$ is a linear function in $\vec{Y}$ alone.

Next we discuss the simplest reaction-diffusion equations that arise in phase changes in cloud modeling [11, 12, 21]. If precipitation through rain and other phase changes such as the formation of ice are ignored, the equations for changes in phase from cloud water vapor to condensed cloud water in bulk microphysics cloud models are described in terms of the following three variables:

$$
\left\{\begin{array}{l}
\text { (i) (potential) temperature } T, \\
\text { (ii) mixing ratio } q_{V} \geq 0 \text { of cloud water vapor, and } \\
\text { (iii) mixing ratio } q_{C} \geq 0 \text { of condensed cloud water, }
\end{array}\right.
$$

where the mixing ratios are computed relative to dry air.

In cloud physics modeling, there is a saturation value of water vapor denoted by $q_{V}^{\text {sat }}$ which is a nonlinear, monotonically increasing function of the temperature and is determined by the Clausius-Clapeyron equation of thermodynamics [12, 21]. If there is any supersaturated cloud vapor with $q_{V}>q_{V}^{\text {sat }}$, this supersaturated vapor condenses to form cloud water and releases latent heat. Conversely, if there is cloud water present with $q_{C}>0$ and the cloud vapor is undersaturated with $q_{V}<q_{V}^{\text {sat }}$, a fraction of the cloud water is converted to cloud vapor with a corresponding evaporative cooling of the environment. In the models considered here, the effect of cloud water on the buoyancy of the flow field is neglected although this can be an important physical effect.

The simplest model in $[11,12]$ incorporating all of these physical phenomena is given by the three convective reaction-diffusion equations

$$
\left\{\begin{array}{l}
D_{t} T=\mathcal{K} \Delta T+q_{0} K q_{C}\left(q_{V}-q_{V}^{\text {sat }}(T)\right) \\
D_{t} q_{V}=\mathcal{K} \Delta q_{V}-K q_{C}\left(q_{V}-q_{V}^{\text {sat }}(T)\right) \quad \text { in } \mathbb{R}^{N} \times(0, \infty), \\
D_{t} q_{C}=\mathcal{K} \Delta q_{C}+K q_{C}\left(q_{V}-q_{V}^{\text {sat }}(T)\right)
\end{array}\right.
$$


with $q_{0}>0$ the latent heat release.

Very natural turbulent mixing problems occur in cloud physics. Consider, for example, an initial state consisting of two disjoint regions $\Omega_{+}$containing dry air and $\Omega_{-}$containing cloudy air. In the dry air region $\Omega_{+}$, the initial data $q_{V, 0}, q_{C, 0}$, and $T_{0}$ satisfy

$$
q_{V, 0}<q_{V}^{\text {sat }}\left(T_{0}\right) \quad \text { and } \quad q_{C, 0}=0 \quad \text { in } \Omega_{+},
$$

while in the cloudy air region, $\Omega_{-}$, the initial data satisfy

$$
q_{V, 0}=q_{V}^{\text {sat }}\left(T_{0}\right) \quad \text { and } \quad q_{C, 0} \geq \bar{q}_{C}>0 \quad \text { in } \Omega_{-} .
$$

Introducing as variables the total cloud water content $Q=q_{V}+q_{C}$ and the enthalpy (called the equivalent potential temperature in [11]) $H=T-q_{0} q_{C}$, the equations in (2.5) are equivalent to

$$
\left\{\begin{array}{l}
D_{t} H=\mathcal{K} \Delta H \\
D_{t} Q=\mathcal{K} \Delta Q \\
D_{t} q_{C}=\mathcal{K} \Delta q_{C}+K q_{C}\left(a\left(H, Q, q_{C}\right)-q_{C}\right)
\end{array} \quad \text { in } \mathbb{R}^{N} \times(0, \infty),\right.
$$

with the nonlinear function $a\left(H, Q, q_{C}\right)$ given by

$$
a\left(H, Q, q_{C}\right)=Q-q_{V}^{\text {sat }}\left(H+q_{0} q_{C}\right) .
$$

Thus if we introduce the variables $\vec{Y}=(H, Q)$ and $Z=q_{C}$, the equations for cloud physics in (2.8) and (2.9) have the prototype structural form listed in (1.10).

Finally, we check that the initial data in both regions containing dry and cloudy air are stable equilibria. In the dry air region $\Omega_{+}$, the requirements in (2.6) guarantee a stable equilibrium since we have

$$
\left.q_{C, 0}=0 \quad \text { and } \quad a=q_{V, 0}-q_{V}^{\text {sat }}{ }_{0} T\right)<0 \quad \text { in } \Omega_{+} .
$$

On the other hand, from (2.7) in the cloudy air region $\Omega_{-}$we have

$$
a\left(H_{0}, Q_{0}, q_{C, 0}\right)=q_{C, 0} \text { in } \Omega_{-},
$$

and the linearized reaction term for $q_{C}$ at the equilibrium is given by

$$
\left.\frac{d}{d q_{C}}\right|_{q_{C, 0}} q_{C}\left(a\left(H, Q, q_{C}\right)-q_{C}\right)=-q_{C, 0}\left(1-\left.q_{0} \frac{\partial}{\partial T}\right|_{T_{0}} q_{V}^{\text {sat }}\right) .
$$

Since $q_{V}^{\text {sat }}(T)$ is a monotone increasing function of the temperature $T$, the equilibrium in the cloudy air is stable provided the latent heat $q_{0}$ is sufficiently small. This last requirement is always satisfied for realistic clouds that have extremely small latent heat release $[11,12,21]$. In fact, to a first approximation, one can set $q_{0}=0$ in (2.9) so that the nonlinear function $a$ in (2.8) and (2.9) is a function of only $H$ and $Q$, i.e., $a(H, Q)$. As a matter of fact, in order to satisfy the assumptions of Theorem 3.1 for this model, we will need to make, for technical reasons, the assumption of asymptotically small heat release, i.e., that $q_{0}=\varepsilon q_{0}^{\prime}$. 


\section{The General Fast Reaction/Slow Diffusion Asymptotics}

Here we study the fast reaction/slow diffusion asymptotics for the prototype turbulent reaction-diffusion equations given by (1.10). Following the discussion in Section 2, we consider the system

$$
\left\{\begin{array}{l}
D_{t}^{\varepsilon} \vec{Y}^{\varepsilon}=\varepsilon \mathcal{K} \Delta \vec{Y}^{\varepsilon} \\
D_{t}^{\varepsilon} Z^{\varepsilon}=\varepsilon \mathcal{K} \Delta Z^{\varepsilon}+\varepsilon^{-1} K\left(\vec{Y}^{\varepsilon}, Z^{\varepsilon}\right) Z^{\varepsilon}\left(a\left(\vec{Y}^{\varepsilon}, \varepsilon Z^{\varepsilon}\right)-Z^{\varepsilon}\right)
\end{array} \text { in } \mathbb{R}^{N} \times(0, \infty) .\right.
$$

For the initial data $\left(\vec{Y}_{0}^{\varepsilon}, Z_{0}^{\varepsilon}\right)$ with $\vec{Y}_{0}^{\varepsilon}=\left(Y_{0}^{\varepsilon, 1}, \ldots, Y_{0}^{\varepsilon, m}\right)$ we assume

$$
\left\{\begin{array}{l}
Y_{0}^{\varepsilon, i}(i=1, \ldots, m) \text { and } Z_{0}^{\varepsilon} \text { are bounded functions such that } \\
\vec{Y}_{0}^{\varepsilon}=0 \text { on } \partial \Omega_{+}=\partial \Omega_{-} \text {and } Z_{0}^{\varepsilon}=0 \text { in } \Omega_{-} \text {and } Z_{0}^{\varepsilon}>0 \text { in } \Omega_{+},
\end{array}\right.
$$

and, as $\varepsilon \rightarrow 0$,

$$
\begin{cases}\vec{Y}_{0}^{\varepsilon} \rightarrow \vec{Y}_{0} & \text { locally uniformly in } \mathbb{R}^{N} \\ Z_{0}^{\varepsilon} \rightarrow Z_{0} & \text { locally uniformly in } \mathbb{R}^{N} \text { with } Z_{0}>0 \text { in } \Omega_{+} .\end{cases}
$$

Throughout this section we assume that

$$
K \text { and } a \text { are bounded, continuous functions }
$$

and, for all $(\vec{Y}, Z)$ with $Z \geq 0$,

$$
K(\vec{Y}, Z)(a(\vec{Y}, \varepsilon Z)-Z) \leq K(\vec{Y}, 0) a(\vec{Y}, 0) .
$$

This last assumption is the "usual" K-P-P-type assumption on the reaction. In the first example of the previous section, (3.5) is satisfied if the heat release $q_{0}$ is sufficiently small and $K$ is, for example, $C^{1}$. In fact, interesting new extinction phenomena happen in this system for larger heat release [14, 24]. In the second example, it follows from the assumption that $a$ is increasing with respect to $q_{C}$. Notice that for this assumption it is not necessary to assume $q_{0}=\varepsilon q_{0}^{\prime}$.

As far as the vector field $\vec{V}^{\varepsilon}$ is concerned, throughout this section we assume that

$$
\left\{\begin{array}{l}
\bar{V} \text { and } v \text { are bounded and Lipschitz-continuous } \\
\text { with respect to their arguments, and }\langle v\rangle=0 \text { and } \operatorname{div} v=0 .
\end{array}\right.
$$

Before we state the result, we remark that the special form of $a$ is assumed here for technical reasons which we identify at the appropriate place. We do not know at this moment, however, whether it can be relaxed. Our main result, which, as should be evident to the reader, is a more precise and general statement of Theorem 1.1 from the introduction, is the following:

THEOREM 3.1 Assume (3.2)-(3.6). Then, as $\varepsilon \rightarrow 0, \vec{Y}^{\varepsilon} \rightarrow \vec{Y}^{0}$ locally uniformly in $\mathbb{R}^{N} \times(0, \infty)$, where $\vec{Y}^{0}$ evolves by

$$
\vec{Y}_{t}^{0}+\bar{V}(x, t) \cdot D \vec{Y}^{0}=0 \quad \text { in } \mathbb{R}^{N} \times(0, \infty) .
$$


Moreover, $Z^{\varepsilon}=\exp \left(\varepsilon^{-1}\left(W^{0}+o(1)\right)\right)$, where $W^{0}$ is the unique viscosity solution of the variational inequality

$$
\left\{\begin{array}{l}
\max \left(W_{t}^{0}+H\left(D W^{0}, x, t\right)-K\left(Y^{0}, 0\right) a\left(Y^{0}, 0\right), W^{0}\right)=0 \quad \text { in } \mathbb{R}^{N} \times(0, \infty) \\
W^{0}= \begin{cases}-\infty & \text { in } \Omega_{-} \times\{0\} \\
0 & \text { in } \Omega_{+} \times\{0\} .\end{cases}
\end{array}\right.
$$

For each $p \in \mathbb{R}^{N}, H(p, x, t)$ is the unique "nonlinear" eigenvalue corresponding to the cell problem

$$
w_{\tau}+(\bar{V}(x, t)+v(y, \tau)) \cdot\left(p+D_{y} w\right)-\mathcal{K}\left|p+D_{y} w\right|^{2}=H(p, x, t) .
$$

Moreover, as $\varepsilon \rightarrow 0, Z^{\varepsilon} \rightarrow a\left(\vec{Y}^{0}, 0\right)$, locally uniformly in $\left\{a\left(\vec{Y}^{0}, 0\right)>0\right\} \cap\left(\mathbb{R}^{N} \times\right.$ $(0, \infty))$ and $Z^{\varepsilon} \rightarrow 0$, locally uniformly, in $\left\{a\left(\vec{Y}^{0}, 0\right)<0\right\} \cap\left(\mathbb{R}^{N} \times(0, \infty)\right)$. Finally, the set $\left\{a\left(\vec{Y}^{0}, 0\right)=0\right\}$ propagates with normal velocity $-\bar{V}$.

The proof of the theorem consists of a number of steps, which are presented below in the form of propositions and lemmas.

We begin with the proof of the first part of Theorem 3.1, namely, the characterization of the limiting behavior, as $\varepsilon \rightarrow 0$, of the vector $\vec{Y}^{\varepsilon}$. Since each component of $\vec{Y}^{\varepsilon}$ evolves independently of the others, it clearly suffices to study the asymptotics, as $\varepsilon \rightarrow 0$, of the solution $w^{\varepsilon}$ of the advection-diffusion equation

$$
\begin{cases}D_{t}^{\varepsilon} w^{\varepsilon}=\varepsilon \mathcal{K} \Delta w^{\varepsilon} & \text { on } \mathbb{R}^{N} \times(0, \infty) \\ w^{\varepsilon}=w_{0}^{\varepsilon} & \text { in } \mathbb{R}^{N} \times\{0\}\end{cases}
$$

where

$$
D_{t}^{\varepsilon}=\partial_{t}+\left(\bar{V}(x, t)+\vec{v}\left(\varepsilon^{-\alpha} x, \varepsilon^{-\alpha} t\right)\right) \cdot D,
$$

with $\bar{V}$ and $\bar{v}$ satisfying (3.6). For the initial datum $w_{0}^{\varepsilon}$ we assume that

$$
\left\{\begin{array}{l}
w_{0}^{\varepsilon} \text { is bounded, uniformly continuous, and, as } \varepsilon \rightarrow 0, w_{0}^{\varepsilon} \rightarrow w_{0} \\
\text { locally uniformly in } \mathbb{R}^{N} \backslash\left(\Omega_{+} \cup \Omega_{-}\right), \text {with } w_{0} \text { a bounded function. }
\end{array}\right.
$$

In a later publication we will analyze the situation where $w_{0}^{\varepsilon}$ depends on more scales.

We have the following:

Proposition 3.2 Assume (3.6) and (3.11). Then, as $\varepsilon \rightarrow 0, w^{\varepsilon} \rightarrow w$, locally uniformly in $\mathbb{R}^{N} \times(0, T)$, for all $T>0$, where $w$ is the solution of

$$
\begin{cases}w_{t}+\bar{V}(x, t) \cdot D w=0 & \text { in } \mathbb{R}^{N} \times(0, \infty) \\ w=w_{0} & \text { on } \mathbb{R}^{N} \times\{0\} .\end{cases}
$$

Before we present the proof, we remark that the fact that the diffusion satisfies $\mathcal{K}>0$ is essential in this result. As remarked in the introduction when $\mathcal{K}=0$, one encounters different phenomena. This is the topic of Section 4. 
There are various ways to prove this proposition. Here we present an argument based on viscosity solutions and the perturbed test function method introduced in Evans [7] (see also [17]). The arguments are by now more or less classical, so below we only present a brief sketch.

PROOF: 1. Since the maximum principle yields that

$$
\sup _{\varepsilon>0}\left\|w^{\varepsilon}\right\|_{L^{\infty}\left(\mathbb{R}^{N} \times[0, \infty)\right)}<\infty,
$$

we may define the half-relaxed limits

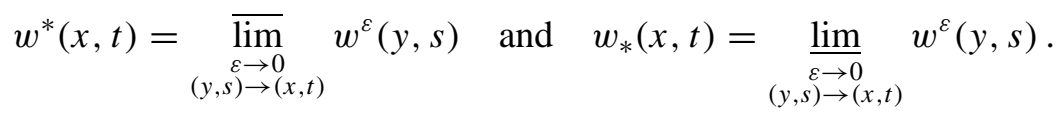

We obviously have

$$
w_{*} \leq w^{*} .
$$

2. Assume that, for a smooth function $\varphi, w^{*}-\phi$ attains a strict local maximum at $\left(x_{0}, t_{0}\right)$. Let $\psi\left(y, \tau ; x_{0}, t_{0}\right)$ be the smooth space-time periodic solution of $\psi_{\tau}+\left(Y\left(x_{0}, t_{0}\right)+v(y, \tau)\right) \cdot D_{y} \psi=\mathcal{K} \varepsilon^{1-\alpha} \Delta_{y} \psi-v(y, \tau) \cdot p \quad$ in $\mathbb{R}^{N} \times \mathbb{R}$.

The existence of such $\psi$ follows easily from a Fredholm's alternative-type argument, in view of the assumption that $\langle v\rangle=0$ and $\operatorname{div} v=0$. Then

$$
w^{\varepsilon}(x, t)-\left(\phi(x, t)+\varepsilon \psi\left(\varepsilon^{-\alpha} x, e^{-\alpha} t, x_{0}, t_{0}\right)\right)
$$

attains a local maximum at $\left(x_{0}, t_{0}\right)$ and $\left(x_{\varepsilon}, t_{\varepsilon}\right) \rightarrow\left(x_{0}, t_{0}\right)$ as $\varepsilon \rightarrow 0$. Applying the maximum principle to $w^{\varepsilon}$, we find that at $\left(x_{\varepsilon}, t_{\varepsilon}\right)$

$$
\phi_{t}+\bar{v} \cdot D \phi \leq o(1) .
$$

Letting $\varepsilon \rightarrow 0$, we conclude that at $\left(x_{0}, t_{0}\right)$

$$
\phi_{t}+\bar{v} \cdot D \phi \leq 0
$$

i.e., that $w^{\varepsilon}$ is a subsolution of (3.12).

3. A similar argument shows that $w_{*}$ is a supersolution of (3.12).

4. The conclusion follows from (3.13) and the fact that (3.12) admits to a unique continuous solution and a comparison principle.

Remark. If $\alpha=1$ in (0.4), a similar result holds, the only difference being that $w_{t}+\bar{V} \cdot D w=0$ in (3.12) is replaced by $w_{t}+\bar{V} \cdot D w=\mathcal{K} \Delta w$.

Proposition 3.2 and the continuity of $a$ imply that as $\varepsilon \rightarrow 0$,

$$
a\left(\vec{Y}^{\varepsilon}, \varepsilon Z^{\varepsilon}\right) \rightarrow a(\vec{Y}, 0) \quad \text { locally uniformly in } \mathbb{R}^{N} \times(0, \infty)
$$

with $A(x, t)=a(\vec{Y}(x, t), 0)$ solving

$$
\begin{cases}A_{t}+\bar{V} \cdot D A=0 & \text { in } \mathbb{R}^{N} \times(0, \infty) \\ A=a\left(Y_{0}, 0\right) & \text { on } \mathbb{R}^{N} \times\{0\}\end{cases}
$$


provided we establish an $L^{\infty}$-bound on $Z^{\varepsilon}$. This is the object of the following:

LEMMA 3.3 For every $T>0$ there exists a positive constant $C=C(T)>0$ such that

$$
0 \leq Z^{\varepsilon} \leq C \quad \text { in } \mathbb{R}^{N} \times[0, T) .
$$

ProOF: 1 . Since $Z^{\varepsilon} \geq 0$ on $\mathbb{R}^{N} \times\{0\}$, the lower bound is an immediate consequence of the maximum principle.

2. Fix $\beta>0$ and define $Q^{\varepsilon}=\left(Z^{\varepsilon}-\beta\right)_{+}$. It follows that

$$
\begin{aligned}
D_{t}^{\varepsilon} Q^{\varepsilon} \leq \varepsilon \mathcal{K} \Delta Q^{\varepsilon}+\varepsilon^{-1} K\left(\vec{Y}^{\varepsilon}, Z^{\varepsilon}\right) Z^{\varepsilon}\left(a\left(\vec{Y}^{\varepsilon}, \varepsilon Z^{\varepsilon}\right)-Z^{\varepsilon}\right) \mathbb{1}_{\left[Z^{\varepsilon}>\beta\right]} & \text { in } \mathbb{R}^{N} \times(0, \infty),
\end{aligned}
$$

where $\mathbb{1}_{A}$ denotes the characteristic function of the set $A$.

3. Choose $\beta=\max \left\{\left\|a\left(\vec{Y}^{\varepsilon}, \varepsilon Z^{\varepsilon}\right)\right\|_{L^{\infty}\left(\mathbb{R}^{N} \times[0, \infty)\right)},\left\|Z_{0}^{\varepsilon}\right\|_{L^{\infty}\left(\mathbb{R}^{N}\right)}\right\}$. Then

$$
K\left(\vec{Y}^{\varepsilon}, Z^{\varepsilon}\right) Z^{\varepsilon}\left(a\left(\vec{Y}^{\varepsilon}, \varepsilon Z^{\varepsilon}\right)-Z^{\varepsilon}\right) 1_{\left[Z^{\varepsilon}>\beta\right]} \leq 0 \quad \text { in } \mathbb{R}^{N} \times(0, \infty),
$$

and hence

$$
\begin{cases}D_{t}^{\varepsilon} Q^{\varepsilon} \leq \varepsilon \mathcal{K} \Delta Q^{\varepsilon} & \text { in } \mathbb{R}^{N} \times(0, \infty) \\ Q^{\varepsilon} \leq 0 & \text { on } \mathbb{R}^{N} \times\{0\} .\end{cases}
$$

It follows that

$$
Q^{\varepsilon} \leq 0 \quad \text { in } \mathbb{R}^{N} \times[0, \infty),
$$

hence the claim.

The next lemma represents the first step towards understanding the limiting behavior of $Z^{\varepsilon}$.

LEMMA 3.4 For each $\delta>0$, and uniformly on compact subsets of $\{A>0\}$ (respectively, $\{A<0\})$ where A solves (3.15),

$$
\left.\lim _{\varepsilon \rightarrow 0}\left(Z^{\varepsilon}-(A+\delta)\right)_{+}=0 \quad \text { (respectively, } \lim _{\varepsilon \rightarrow 0}\left(Z^{\varepsilon}-\delta\right)_{+}=0\right) .
$$

ProOF: 1. Define $Q^{\varepsilon}=\left(Z^{\varepsilon}-\delta\right)_{+}$. Following the calculation of Lemma 3.3, we find that

$D_{t}^{\varepsilon} Q^{\varepsilon} \leq \varepsilon \mathcal{K} Q^{\varepsilon}+\varepsilon^{-1} K\left(\vec{Y}^{\varepsilon}, Z^{\varepsilon}\right) Z^{\varepsilon}\left(a\left(\vec{Y}^{\varepsilon}, \varepsilon Z^{\varepsilon}\right)-Z^{\varepsilon}\right) \mathbb{1}_{\left[Z^{\varepsilon} \geq \delta\right]} \quad$ in $\mathbb{R}^{N} \times(0, \infty)$.

2. It is enough to consider the behavior of $Z^{\varepsilon}$ in cylinders $\mathcal{C}$ of the form

$$
\mathcal{C}=\left\{\left|x-x_{0}\right|<R, t \in(0, T]\right\} \subset\{A<0\},
$$

where, for $\varepsilon$ sufficiently small depending on $R$ and $T$,

$$
a\left(\vec{Y}^{\varepsilon}, \varepsilon Z^{\varepsilon}\right) \leq A+\delta .
$$

It follows that

$K\left(\vec{Y}^{\varepsilon}, Z^{\varepsilon}\right) Z^{\varepsilon}\left(a\left(\vec{Y}^{\varepsilon}, \varepsilon Z^{\varepsilon}\right)-Z^{\varepsilon}\right) \leq-\delta K\left(\vec{Y}^{\varepsilon}, Z^{\varepsilon}\right)\left(Z^{\varepsilon}-\delta\right) \quad$ in $\mathcal{C} \cap\left[Z^{\varepsilon}>\delta\right]$, 
and hence

$$
D_{t}^{\varepsilon} Q^{\varepsilon} \leq \varepsilon \mathcal{K} \Delta Q^{\varepsilon}-\varepsilon^{-1} \delta K\left(\vec{Y}^{\varepsilon}, Z^{\varepsilon}\right) Q^{\varepsilon} \quad \text { in } \mathcal{C} .
$$

3. Let $\phi$ be the smooth solution of

$$
\begin{cases}\Delta \phi=-1 & \text { in }\left\{\left|x-x_{0}\right|<R\right\} \\ \phi=0 & \text { on }\left\{\left|x-x_{0}\right|=R\right\}\end{cases}
$$

and define, for $\lambda>0$,

$$
\widetilde{Q}^{\varepsilon}(x, t)=C_{\delta} \exp \left(-\varepsilon^{-1} \lambda \delta t \phi(x)\right)
$$

where $C_{\delta}$ is such that

$$
Q^{\varepsilon} \leq C_{\delta} \equiv(C-\delta)_{+} \text {on the parabolic boundary of } \mathcal{C} .
$$

For $\lambda$ sufficiently small we have

$$
\begin{aligned}
D_{t}^{\varepsilon} \widetilde{Q}^{\varepsilon}-\varepsilon \mathcal{K} \Delta \widetilde{Q}^{\varepsilon}+\varepsilon^{-1} K\left(\vec{Y}^{\varepsilon}, Z^{\varepsilon}\right) \delta \widetilde{Q}^{\varepsilon} \\
=\varepsilon^{-1} \widetilde{Q}^{\varepsilon}\left[\delta\left(K\left(\vec{Y}^{\varepsilon}, Z^{\varepsilon}\right)-\lambda \phi\right)-\lambda t \delta V_{\varepsilon} \cdot D \phi\right. \\
\left.\quad+\varepsilon \delta \lambda t \mathcal{K} \Delta \phi-\lambda^{2} t^{2} \delta^{2} \mathcal{K}|D \phi|^{2}\right] \geq 0 .
\end{aligned}
$$

Hence

$$
Q^{\varepsilon}(x, t) \leq C_{\delta} \exp \left(-\varepsilon^{-1} \lambda \delta t \phi(x)\right) \quad \text { in } \mathcal{C} .
$$

The conclusion follows from the fact that, as $\varepsilon \rightarrow 0$,

$$
\exp \left(-\varepsilon^{-1} \lambda \delta t \phi(x)\right) \rightarrow 0 \quad \text { if }\left|x-x_{0}\right|<R .
$$

4. Consider a cylinder of the form

$$
\mathcal{C}=\left\{\left|x-x_{0}\right|<R, t \in(0, T]\right\} \subset\{A>0\},
$$

where we may assume that, for sufficiently small $\varepsilon>0$,

$$
a\left(\vec{Y}^{\varepsilon}, \varepsilon Z^{\varepsilon}\right) \leq A\left(x_{0}, t_{0}\right)+\delta \text { in } \mathcal{C}
$$

and define

$$
Q^{\varepsilon}=\left(Z^{\varepsilon}-\left(A\left(x_{0}, t_{0}\right)+\delta\right)\right)_{+} .
$$

Observe then that there exists $\sigma>0$ such that

$$
\begin{aligned}
Z^{\varepsilon}\left(a\left(\vec{Y}^{\varepsilon}, \varepsilon Z^{\varepsilon}\right)-Z^{\varepsilon}\right) & \leq-\sigma\left(Z^{\varepsilon}-a\left(Y^{\varepsilon}-a\left(Y^{\varepsilon}, \varepsilon Z^{\varepsilon}\right)\right)\right) \\
& \leq-\sigma\left(Z^{\varepsilon}-\left(A\left(x_{0}, t_{0}\right)+\delta\right)\right) \text { on } \mathcal{C} .
\end{aligned}
$$

It follows that

$$
D_{t}^{\varepsilon} Q^{\varepsilon}-\varepsilon \mathcal{K} \Delta Q^{\varepsilon}+\varepsilon^{-1} K\left(\vec{Y}^{\varepsilon}, Z^{\varepsilon}\right) \sigma Q^{\varepsilon} \leq 0 \text { in } \mathcal{C} .
$$

Arguing as in Step 3 above, we conclude.

As an immediate consequence of Lemma 3.4, we have the following:

Proposition 3.5 As $\varepsilon \rightarrow 0, Z^{\varepsilon} \rightarrow 0$ uniformly on compact subsets of $\{A<0\}$. 
PROOF: Lemma 3.4 implies that $\varlimsup_{\varepsilon \rightarrow 0} Z^{\varepsilon} \leq 0$ uniformly on compact subsets of $\{A<0\}$. Since $Z^{\varepsilon} \geq 0$, the conclusion follows.

We continue the analysis of the asymptotic behavior of $Z^{\varepsilon}$ by performing the classical logarithmic change of variables,

$$
Z^{\varepsilon}=\exp \left(\varepsilon^{-1} W^{\varepsilon}\right) .
$$

It follows that $W^{\varepsilon}$ solves the initial value problem

$$
\left\{\begin{aligned}
& D_{t}^{\varepsilon} W_{t}^{\varepsilon}= \varepsilon \mathcal{K} \Delta W^{\varepsilon}+\mathcal{K}\left|D W^{\varepsilon}\right|^{2}+K\left(\overrightarrow{Y^{\varepsilon}}, Z^{\varepsilon}\right)\left(a\left(\vec{Y}^{\varepsilon}, \varepsilon Z^{\varepsilon}\right)-Z^{\varepsilon}\right)=0 \\
& \quad \text { in } \mathbb{R}^{N} \times(0, \infty) \\
& W^{\varepsilon}= \begin{cases}-\infty & \text { in } \Omega_{-} \times\{0\} \\
\varepsilon \ln Z_{0}^{\varepsilon} & \text { in } \bar{\Omega}_{+} \times\{0\} .\end{cases}
\end{aligned}\right.
$$

The following proposition is proved as theorem 1.1 in [17]:

Proposition 3.6 As $\varepsilon \rightarrow 0, W^{\varepsilon} \rightarrow W$, locally uniformly in $\mathbb{R}^{N} \times(0, \infty)$, where $W$ is the unique viscosity solution of the variational inequality (3.8), with $H$ defined in the statement of Theorem 3.1. Moreover, as $\varepsilon \rightarrow 0, Z^{\varepsilon} \rightarrow A$ uniformly and exponentially fast in compact subsets of int $\{W=0\} \cap\{A>0\}$ and $Z^{\varepsilon} \rightarrow 0$ uniformly in compact subsets of $\{Z<0\}$ and exponentially fast.

It follows from Propositions 3.5 and 3.6 that the set $\{A=0\}$ plays an essential role in the analysis of the behavior of $Z^{\varepsilon}$ in the limit $\varepsilon \rightarrow 0$. Since $A$ satisfies (3.15), the following proposition is immediate:

Proposition 3.7 The set $\{A=0\}$ moves with normal velocity $V=\bar{V}(x, t) \cdot n$, $n$ denoting the outward normal to $\{A>0\}$.

The set $\{A>0\}$ is not smooth, so the statement of Proposition 3.7 has to be interpreted in the weak sense using the level set approach. Notice that (3.15) is an equation to which this theory applies. (See [22] for an overview.)

To be able to completely characterize the limiting behavior of $Z^{\varepsilon}$ and thus conclude the proof of Theorem 3.1, we need to understand the relationship between the sets $\{A=0\}$ and $\partial\{W<0\}$. This is the topic of the next proposition.

Proposition 3.8 We have $\{A>0\} \subset \operatorname{int}\{W=0\}$.

PROOF: 1. Arguing by contradiction, we assume that there exist $\left(x_{0}, t_{0}\right) \in$ $\mathbb{R}^{N} \times(0, \infty)$ such that

$$
A\left(x_{0}, t_{0}\right)>0 \text { and } W\left(x_{0}, t_{0}\right)<0 .
$$

2. Since

$$
H(p, x, t)=\bar{V}(x, t) \cdot p-\mathcal{K}\left(|p|^{2}+\left\langle\left|D_{y} w\right|^{2}\right\rangle\right)
$$

in a neighborhood $\mathcal{N}$ of $\left(x_{0}, t_{0}\right)$, we have

$$
W_{t}+\bar{V} \cdot D W \geq K\left(\vec{Y}^{0}, 0\right) A .
$$


If $A \geq 0$, it follows that

$$
D_{t} W \geq 0
$$

i.e., along the trajectory $\dot{x}=\bar{V}(x(t), t)$,

$$
\frac{d}{d t} W(x(t), t) \geq 0 .
$$

3. Proposition 3.7 and assumption (3.2) yield that $A>0$ along a neighborhood of any such trajectory passing through $\left(x_{0}, t_{0}\right)$, hence

$$
0>W\left(x\left(t_{0}\right), t_{0}\right) \geq W\left(X^{-1}\left(x\left(t_{0}\right), 0\right), 0\right)=0 .
$$

The proof of Theorem 3.1 is now complete.

\section{The Zero-Diffusion Case}

We consider here the behavior, as $\varepsilon \rightarrow 0$, of the system

$$
\begin{cases}D_{t}^{\varepsilon} Y^{\varepsilon}=0, D_{t}^{\varepsilon} Z^{\varepsilon}=\varepsilon^{-1} K Z^{\varepsilon}\left(Y^{\varepsilon}-Z^{\varepsilon}\right) & \text { in } \mathbb{R}^{2} \times(0, \infty) \\ Y^{\varepsilon}=Y_{0}, Z^{\varepsilon}=Z_{0}^{\varepsilon} & \text { on } \mathbb{R}^{2} \times\{0\},\end{cases}
$$

where

$$
Z_{0}^{\varepsilon}= \begin{cases}0 & \text { in } \Omega_{-} \times\{0\} \\ Z_{0}>0 & \text { in } \bar{\Omega}_{+} \times\{0\}\end{cases}
$$

and

$$
Y_{0}>0 \text { in } \Omega_{+}, \quad Y_{0}<0 \text { in } \Omega_{-}, \quad \text { and } \quad Y_{0} \leq Z_{0}^{\varepsilon} .
$$

Here

$$
D_{t}^{\varepsilon}=\partial_{t}+\left(\bar{V}(x, t)+v\left(\varepsilon^{-1} x, \varepsilon^{-1} t\right)\right) \cdot D .
$$

The detailed behavior of (4.1) in the limit $\varepsilon \rightarrow 0$ is complicated. Weinan $\mathrm{E}$ [3] studied the limit behavior of the $Y^{\varepsilon}$ equation (see also [23]) and showed that it depends on the detailed properties of the vector field $V^{\varepsilon}$.

In what follows we borrow the example of [3] and show that the limiting behavior of (4.1) is not governed by the large-scale flow. To this end, we assume that

$$
\bar{V}=\left(\begin{array}{l}
\bar{a}_{2} \\
\bar{a}_{1}
\end{array}\right) \quad\left(\text { with } \bar{a}_{1}, \bar{a}_{2} \in \mathbb{R} \backslash\{0\}\right),
$$

and

$$
v\left(x_{1}, x_{2}\right)=\left(-\frac{\partial \psi}{\partial x_{2}}, \frac{\partial \psi}{\partial x_{1}}\right)
$$

where

$$
\psi \text { is periodic in }[0,1]^{2} \text { and has mean } 0 .
$$


The following theorem is proved in [3]:

THEOREM 4.1 Assume that $\bar{a}_{1} / \bar{a}_{2}$ is irrational and that $Y_{0}^{\varepsilon}$ is independent of $\varepsilon$. Then there exists $\mu \in(0,1)$ such that, as $\varepsilon \rightarrow 0, Y^{\varepsilon} \rightarrow Y$ in $L^{2}$, where

$$
\begin{cases}Y_{t}+V^{*} \cdot D Y=\left(V^{*}-\bar{V}\right) D Y_{0} & \text { in } \mathbb{R}^{2} \times(0, \infty) \\ Y=Y_{0} & \text { on } \mathbb{R}^{2} \times\{0\},\end{cases}
$$

where $V^{*}=(1-\mu)^{-1} \bar{V}$.

The equation derived in [3] appears to be different from (4.8). A straightforward calculation, however, using (5.12) and (5.13) of [3] yields (4.8).

Next we investigate the behavior of $Z^{\varepsilon}$. As in Section 3, it follows that there exists $C_{T}>0$ such that

$$
0 \leq Z^{\varepsilon} \leq C_{T} \quad \text { on } \mathbb{R}^{2} \times[0, T] .
$$

Moreover, since clearly $Y^{\varepsilon}$ also solves the equation satisfied by $Z^{\varepsilon}$ and $Y \leq Z^{\varepsilon}$ on $\mathbb{R}^{2} \times\{0\}$,

$$
Y^{\varepsilon} \leq Z^{\varepsilon} \quad \text { on } \mathbb{R}^{2} \times[0, \infty) .
$$

Combining (4.9) and (4.10) we find

$$
\max \left(Y^{\varepsilon}, 0\right) \leq Z^{\varepsilon} \quad \text { in } \mathbb{R}^{2} \times[0, \infty) .
$$

Let $v_{x, t}$ be the Young measure associated with $Z^{\varepsilon}$ (see $[3,23]$ for the exact definition). In view of (4.11) and the strong convergence of the $Y^{\varepsilon}$, we obtain that

the Young measure $v_{x, t}$ is supported in $[\max (Y(x, t), 0), C]$.

It also follows, letting $\varepsilon \rightarrow 0$ in the equation satisfied by $Z^{\varepsilon}$, that

$$
\int z(Y(x, t)-z) d v_{x, t}(z)=0 .
$$

Combining (4.12) and (4.13) we find

$$
v_{x, t}(z)= \begin{cases}\delta(z) & \text { if } Y(x, t)<0 \\ \delta(z-Y(x, t)) & \text { if } Y(x, t)>0\end{cases}
$$

and

$$
Z(x, t)=\int z d v_{x, t}(z)= \begin{cases}0 & \text { if } Y(x, t)<0 \\ Y(x, t) & \text { if } Y(x, t)>0 .\end{cases}
$$

We summarize the above discussion in the following:

THEOREM 4.2 Assume (4.2), (4.3), and (4.4), and the assumptions of Theorem 4.1. Then, as $\varepsilon \rightarrow 0, Z^{\varepsilon} \rightarrow Z$ in $L^{2}$ with $Z$ given by (4.14). 
Assume next that

$$
Y_{0}(x)=y_{0}(x \cdot \beta) \quad \text { with } s y_{0}(s)>0 \text { for } s \neq 0 .
$$

A straightforward computation then yields that the solution $Y$ of (4.8) is given by

$$
\begin{aligned}
Y(x, t) & =\left(V^{*} \beta\right)^{-1}\left[\left(V^{*}-\bar{V}\right) \beta Y_{0}(x)+\bar{V} \beta Y_{0}\left(x-V^{*} \beta t\right)\right] \\
& =\mu Y_{0}(x)+\frac{1}{1-\mu} Y_{0}\left(x-V^{*} \beta t\right) .
\end{aligned}
$$

It is immediate to check that the set $\{Y=0\}$ does not propagate with normal velocity equal to the large-scale flow $\bar{V}$.

Next we consider the case that

$$
Y_{0} \geq Z_{0}^{\varepsilon} \geq 0 \text { and } Y_{0}>0 .
$$

It follows that

$$
Y^{\varepsilon}>0 \quad \text { and } \quad Y^{\varepsilon} \geq Z^{\varepsilon} \quad \text { in } \mathbb{R}^{2} \times[0, \infty) .
$$

Notice that the above assumptions put this case in the inviscid K-P-P regime. The asymptotics of the viscous K-P-P equation were studied, as already remarked in the introduction, in $[8,9,10]$ in the absence of oscillations and in [17] for $V^{\varepsilon}$ as in (3.6) and $\alpha \in(0,1]$.

Here we remark that the asymptotics of $Z^{\varepsilon}$ again are not governed by the mean flow, as can be easily seen from the following example: For simplicity, we consider the situation where $Y_{0} \equiv 1$ and $K=1$. It follows that

$$
Y^{\varepsilon} \equiv Y \equiv 1 \text {. }
$$

Let $W^{\varepsilon}=\exp \left[\varepsilon^{-1} Z^{\varepsilon}\right]$. A straightforward computation yields

$$
D_{t}^{\varepsilon} W^{\varepsilon}=Y-Z^{\varepsilon} \quad \text { in } \mathbb{R}^{2} \times(0, \infty),
$$

and hence

$$
D_{t}^{\varepsilon} W^{\varepsilon} \leq 1 \quad \text { in } \mathbb{R}^{2} \times(0, \infty) .
$$

It follows that

$$
W^{\varepsilon} \leq W^{A, \varepsilon} \quad \text { in } \mathbb{R}^{2} \times(0, \infty),
$$

where, for $A<0$,

$$
\begin{cases}D_{t}^{\varepsilon} W^{A, \varepsilon}=1 & \text { on } \mathbb{R}^{2} \times(0, \infty) \\ W^{A, \varepsilon}=W_{0}^{A} & \text { on } \mathbb{R}^{2} \times\{0\},\end{cases}
$$

where $W_{0}^{A}(x)=w_{0}(x \cdot \beta)$ with

$$
w_{0}=A \text { for } x<0 \text { and } w_{0}=0(\varepsilon) \text { for } x>0 .
$$

It follows that

$$
\varlimsup W^{\varepsilon} \leq W^{A},
$$

where $W^{A}$ solves (4.8). The claim now follows. 


\section{Turbulent Mixing with Slow Reaction and Slow Diffusion}

We begin by summarizing the hypotheses describing the slow reaction/slow diffusion limiting behavior for the equations in (1.10) already discussed in the paragraph above (1.13) in the introduction. We require

$$
\text { (5.1) } \mathcal{K}=\varepsilon \mathcal{K}^{\prime}, \quad K=\varepsilon K^{\prime}, \quad \bar{V}=\varepsilon \bar{V}^{\prime}(x, \varepsilon t), \quad \text { and } \quad v=v\left(\varepsilon^{-1} x, \varepsilon^{-1} t\right)
$$

for the slow reaction/slow diffusion limit. Thus by introducing the longer time scale $t^{\prime}=\varepsilon t$ and utilizing (5.1), the turbulent reaction-diffusion equations from (1.10) become

$$
\begin{aligned}
& \vec{Y}_{t}^{\varepsilon}+\left[\bar{V}(x, t)+\varepsilon^{-1} v\left(\varepsilon^{-1} x, \varepsilon^{-2} t\right)\right] \cdot D \vec{Y}^{\varepsilon}=\mathcal{K} \Delta \vec{Y}^{\varepsilon}, \\
& Z_{t}^{\varepsilon}+\bar{V}(x, t)+\varepsilon^{-1} v\left(\varepsilon^{-1} x, \varepsilon^{-2} t\right) \cdot D Z^{\varepsilon}= \\
& \quad \mathcal{K} \Delta Z^{\varepsilon}+K\left(Y^{\varepsilon}, Z^{\varepsilon}\right)\left(Z^{\varepsilon}\left(a\left(X^{\varepsilon}, Z^{\varepsilon}\right)-Z^{\varepsilon}\right)\right),
\end{aligned}
$$

with all of the primes dropped in (5.2) for notational convenience. To keep the exposition simple, we consider (5.2) with smooth, bounded initial data.

The advection-diffusion equations for $\vec{Y}^{\varepsilon}$ in (5.2) have the standard form for applying the classical homogenization theory $[1,15,16,20]$. The concise presentation of the material in [15, pp. 246-257] is a convenient reference point for the facts summarized below.

We introduce the linear operator with periodic coefficients

$$
Q^{(\text {per })}=\partial_{\tau}+\vec{v}(\xi, \tau) \cdot D_{\xi}-\mathcal{K} \Delta_{\xi} \text {. }
$$

Then $\vec{Y}^{\varepsilon}$ has the formal asymptotic multiple scale expansion

$$
\vec{Y}^{\varepsilon}=\vec{Y}(x, t)+\left.\varepsilon \vec{X}(\xi, \tau)\right|_{\substack{\xi=\varepsilon^{-1} x \\ \tau=\varepsilon^{-2} t}} \cdot D \vec{Y}+\left.\varepsilon^{2} \vec{Y}_{2}(\xi, \tau, x, t)\right|_{\substack{\xi=\varepsilon^{-1} x \\ \tau=\varepsilon^{-2} t}}+O\left(\varepsilon^{3}\right),
$$

where $\vec{X}(\xi, \tau)$ is the unique periodic mean-zero solution to the cell problem

$$
Q^{\text {(per) }} \vec{X}=-v(\xi, \tau)
$$

By inserting the formal asymptotic expansion on the right-hand side of (5.3) into the advection-diffusion equations in (5.2), the terms of order $O\left(\varepsilon^{-2}\right)$ and $O\left(\varepsilon^{-1}\right)$ automatically vanish satisfied, while the terms of order $O\left(\varepsilon^{0}\right)$ yield the equation

$$
\begin{aligned}
Q^{(\text {per })} \vec{Y}_{2}= & -\vec{Y}_{t}-\bar{V} \cdot D_{x} \vec{Y}-\mathcal{K} \Delta_{x} \vec{Y}-\vec{v} \cdot D_{x}\left(\vec{X} \cdot D_{x} \vec{Y}\right) \\
& +\left\{2 D_{x} \cdot\left(D_{\xi} \vec{X} \cdot D \vec{Y}\right)\right\}
\end{aligned}
$$

A unique smooth solution $\vec{Y}_{2}$ of the equation in (5.5) exists (by Fredholm's alternative) if and only if the mean of the right-hand side with respect to the $(\xi, \tau)$ variables vanishes. The term in braces in (5.5) always has mean zero, while standard manipulations of this mean for the remaining terms (see [15] for a summary) using the incompressibility of $\bar{V}$ and $v$ yield the homogenized equation

$$
\vec{Y}_{t}+\vec{V} \cdot D \vec{Y}=\mathcal{K} \Delta \vec{Y}+D \cdot\left(\mathcal{K}_{T} \cdot D \vec{Y}\right)
$$


with the effective diffusivity tensor $\mathcal{K}_{T}=\left(\mathcal{K}_{T}^{i j}\right)$ determined by

$$
\mathcal{K}_{T}^{i j}=\left\langle D \vec{X}_{i} \cdot D \vec{X}_{j}\right\rangle,
$$

with $\vec{X}$ satisfying (5.6) and \langle\rangle denoting the periodic mean in $\xi, \tau$. This is readily made rigorous for smooth initial data by the maximum principle [15] with the estimate

$$
\sup _{\substack{0 \leq t \leq T \\ x \in \mathbb{R}^{N}}}\left|\vec{Y}^{\varepsilon}(x, t)-\vec{Y}(x, t)\right| \leq C(T) \varepsilon .
$$

Similar formal asymptotic expansions apply to the equation for $Z^{\varepsilon}$ in (5.2) with chemical source term with

$$
Z^{\varepsilon}=Z(\vec{x}, t)+\left.\varepsilon \vec{X}(\xi, \tau)\right|_{\substack{\xi=\varepsilon^{-1} x \\ \tau=\varepsilon^{-2} t}} \cdot D Z+\varepsilon^{2} Z_{2}+O\left(\varepsilon^{3}\right) .
$$

The terms of order $O\left(\varepsilon^{-2}\right), O\left(\varepsilon^{-1}\right)$ automatically vanish, while the terms of order $O\left(\varepsilon^{0}\right)$ yield the same equation for $Z_{2}$ as in (5.5) with an additional term on the right-hand side due to the reactive sources given by

$$
K(\vec{Y}, Z)(Z(a(\vec{Y}, Z)-Z)) .
$$

In the same fashion as we discussed after (5.5), the mean of the right-hand side of the equation yields the homogenized reaction-diffusion equation

$$
Z_{t}+\vec{V} \cdot D Z=\mathcal{K} \Delta Z+D \cdot\left(\mathcal{K}_{T} \cdot D Z\right)+K(\vec{Y}, Z)(Z(a(\vec{Y}, Z)-Z))
$$

Equations (5.6) and (5.7) describe the limiting behavior in the slow reaction and slow diffusion limit. For brevity we leave the straightforward rigorous derivation of (5.7) based on the maximum principle as an exercise for the interested reader.

The main physical consequence produced by the results here is that the effect of the small-scale periodic turbulence yields a turbulent eddy diffusivity in the homogenized reaction-diffusion equations (5.6) and (5.7). This derivation requires the rather stringent assumptions on the time scales of the reaction and mean flow listed in (5.1). Such types of turbulent diffusivity modeling are often used for numerical modeling in atmosphere/ocean science $[2,11,12,21]$, where, however, such stringent requirements are often not satisfied.

Acknowledgments. The authors thank Anne Bourlioux for her suggestion to explain the general results first for the simplest case, isothermal non-premixed turbulent diffusion flames. The research of Andrew Majda was partially supported by grants from the Army Research Office (ARO DAAG55-98-1-0129), the National Science Foundation (NSF DMS-997-2865), and the Office of Naval Research (ONR N00014-96-1-0043). The research of Panagiotis Souganidis was partially supported by grants from the National Science Foundation and the European TMR programs on "Hyperbolic Conservation Laws" and "Viscosity Solutions and Their Applications." 


\section{Bibliography}

[1] Bensoussan, A.; Lions, J.-L.; Papanicolaou, G. Asymptotic analysis for periodic structures. Studies in Mathematics and Its Applications, 5. North-Holland, Amsterdam-New York, 1978.

[2] Dowey, S. C.; Gover, D. M.; Najjar, R. G. A new coupled one-dimensional biological-physical model for the upper ocean. Deep-Sea Research II 43 (1996), 591-624.

[3] E, W. N. Homogenization of linear and nonlinear transport equations. Comm. Pure Appl. Math. 45 (1992), no. 3, 301-326.

[4] Embid, P. F.; Majda, A. J.; Souganidis, P. E. Effective geometric front dynamics for premixed turbulent combustion with separated velocity scales. Comb. Sci. Tech. 103 (1994), 85-115.

[5] Embid, P. F.; Majda, A. J.; Souganidis, P. E. Comparison of turbulent flame speeds from complete averaging and the $G$-equation. Phys. Fluids 7 (1995), no. 8, 2052-2060.

[6] Embid, P. F.; Majda, A. J.; Souganidis, P. E. Examples and counterexamples for Huygens Principle in premixed combustion. Comb. Sci. Tech. 120 (1996), 273-303.

[7] Evans, L. C. Periodic homogenisation of certain fully nonlinear partial differential equations. Proc. Roy. Soc. Edinburgh Sect. A 120 (1992), no. 3-4, 245-265.

[8] Evans, L. C.; Souganidis, P. E. A PDE approach to geometric optics for certain semilinear parabolic equations. Indiana Univ. Math. J. 38 (1989), no. 1, 141-172.

[9] Freĭdlin, M. Functional integration and partial differential equations. Annals of Mathematics Studies, 109. Princeton University Press, Princeton, N.J., 1985.

[10] Frě̆dlin, M. Limit theorems for large deviations and reaction-diffusion equations. Ann. Probab. 13 (1985), no. 3, 639-675.

[11] Grabowski, W. Cumulus entrainment, fine scale mixing, and buoyancy reversal. Q. J. Roy. Meteorol. Soc. 119 (1993), 935-956.

[12] Grabowski, W.; Smolarkiewicz, P. Two-time level semi-Lagrangian modeling of precipitating clouds. Monthly Weather Rev. 124 (1996), 487-497.

[13] Levin, S. The problem of pattern and scale in ecology. Ecology 73 (1992), 1943-1967.

[14] Linan, A.; Williams, F. A. Fundamental aspects of combustion. The Oxford Engineering Science Series, 33. Chapters 3 and 5. Oxford University Press, New York, 1993.

[15] Majda, A.; Kramer, P. R. Simplified models for turbulent diffusion: theory, numerical modelling, and physical phenomena. Phys. Rep. 314 (1999), no. 4-5, 237-574.

[16] Majda, A. J.; McLaughlin, R. M. The effect of mean flows on enhanced diffusivity in transport by incompressible periodic velocity fields. Stud. Appl. Math. 89 (1993), no. 3, 245-279.

[17] Majda, A. J.; Souganidis, P. E. Large-scale front dynamics for turbulent reaction-diffusion equations with separated velocity scales. Nonlinearity 7 (1994), no. 1, 1-30.

[18] Majda, A. J.; Souganidis, P. E. Bounds on enhanced turbulent flame speeds for combustion with fractal velocity fields. J. Statist. Phys. 83 (1996), 933-954.

[19] Majda, A. J.; Souganidis, P. Flame fronts in a turbulent combustion model with fractal velocity fields. Comm. Pure Appl. Math. 51 (1998), no. 11-12, 1337-1348.

[20] McLaughlin, D. W.; Papanicolaou, G. C; Pironneau, O. R. Convection of microstructure and related problems. SIAM J. Appl. Math. 45 (1985), no. 5, 780-797.

[21] Rogers, R. R.; Yau, M. K. A short course in cloud physics. 3rd ed. International Series in Natural Philosophy, 113. Pergamon, Oxford-New York, 1989.

[22] Souganidis, P. E. Front propagation: theory and applications. Viscosity solutions and applications (Montecatini Terme, 1995), 186-242. Lecture Notes in Mathematics, 1660. Springer, Berlin, 1997.

[23] Tartar, L. Compensated compactness and applications to partial differential equations. Nonlinear analysis and mechanics: Heriot-Watt Symposium, Vol. IV, 136-212. Research Notes in Mathematics, 39. Pitman, Boston-London, 1979. 
[24] Williams, F. A. Combustion theory. 2nd ed. Chapters 3 and 10. Benjamin/Cummings, Menlo Park, Calif., 1985.

ANDREW J. MAJDA

Courant Institute

251 Mercer Street

New York, NY 10012
PANAGIOTIS E. SOUGANIDIS

University of Wisconsin-Madison

Department of Mathematics

480 Lincoln Drive, Room 214

Madison, WI 53706

E-mail: souganid@math.wisc.edu

Received September 1999. 